\title{
OCORRÊNCIA DE OVOS DE Toxocara SPp. EM SOLOS DE PRAÇAS PÚBLICAS DE LAGES, SANTA CATARINA
}

\author{
OCCURRENCE OF Toxocara spp. SOILS OF PUBLIC SQUARES LAGES, SANTA CATARINA
}

\author{
R. M. QUADROS ${ }^{1}$, F. R. LIZ ${ }^{2}$, S. M. T. MARQUES ${ }^{3}$
}

\begin{abstract}
RESUMO
O trabalho teve por objetivo verificar a ocorrência de parasitos de potencial zoonótico em solos de oito praças públicas em três estações do ano, na região urbana de Lages, Santa Catarina. Foram processadas 1602 amostras de solo, 504 de praças centrais e 1098 de praças da periferia urbana, em sete bairros. As análises parasitológicas detectaram somente ovos de Toxocara spp. (0,75\%), distribuídos em: 0,44 na primavera, $0,25 \%$ no verão e $0,06 \%$ no outono, sem apresentar diferença estatística. A Praça Parque Jonas Ramos, no centro da cidade, obteve maior número de amostras positivas $(1,51 \%)$. Conclui-se que a presença deste parasito zoonótico pode apresentar risco à saúde, sobretudo para crianças que fazem destes locais um ambiente lúdico.
\end{abstract}

PALAVRAS-CHAVE: Área de lazer. Helminto. Toxocara. Zoonose.

\section{SUMMARY}

The aim of this study was to assess the occurrence of potentially zoonotic parasites in soils of eight public squares during three seasons of the year, in the urban region of Lages, Santa Catarina, Brazil. A total of 1,602 soil samples were processed, 504 from downtown squares and 1,098 from squares located on the outskirts, in seven neighborhoods. Parasitological tests detected only Toxocara spp. eggs $(0.75 \%)$, distributed as follows: $0.44 \%$ in spring, $0.25 \%$ in summer, and $0.06 \%$ in fall, without any statistical difference. The Parque Jonas Ramos square, located downtown, yielded the largest number of positive samples (1.51\%). In conclusion, the presence of this zoonotic parasite may be a health hazard, especially to children who play in these squares.

KEY-WORDS: Helminth, Leisure area, Toxocara, Zoonotic disease.

\footnotetext{
${ }^{1}$ Médica Veterinária, Dra. e Profa. de Parasitologia e Bióloga, Laboratório de Parasitologia da UNIPLAC e profa. de Doenças Parasitárias do CAV/UDESC. Email.: rosileia18@ hotmail.com

${ }^{2}$ Bióloga, Laboratório de Parasitologia, Faculdade de Biologia da UNIPLAC. Email.: feronconi2@ hotmail.com

${ }^{3}$ Médica Veterinária, PhD, Pesquisadora, Departamento de Patologia Clínica Veterinária, Laboratório de Helmintologia, Faculdade de Veterinária, UFRGS. Email.: smtmuni@ hotmail.com
} 


\section{INTRODUÇÃO}

As infecções parasitárias estão entre os mais frequentes agravos à saúde humana no mundo e podem afetar o equilíbrio nutricional, interferindo, sobretudo na absorção de nutrientes. A população infantil corresponde ao grupo mais exposto devido ao hábito de brincar em contato com o solo que aliados aos distúrbios de perversão do apetite, aumentam a chance de infecção ao ingerir acidentalmente ovos larvados de Toxocara spp. presentes no solo, em fômites e em mãos contaminadas (COELHO et al., 2001). Por outro lado, o crescimento urbano, com o estabelecimento de novas comunidades e conjuntos habitacionais, leva tanto ao aumento da população de cães de estimação como de cães errantes. Do ponto de vista epidemiológico, cães errantes têm um papel importante na contaminação do meio ambiente, uma vez que não recebem tratamento antiparasitário e podem ter fácil acesso a áreas públicas. $\mathrm{O}$ crescente número de cães domiciliados e errantes em todo o Brasil vem contribuindo para uma maior dispersão destes agentes parasitários (SCAINI et al., 2003). Os cães e gatos com acesso aos locais de recreação contaminam o solo, eliminando até 15.000 ovos de parasitos por grama de fezes (ARAÚJO et al., 1999).

As áreas públicas e locais de recreação como os parques e playgrounds podem ser fontes de infecção de helmintos para animais e pessoas. A presença de ovos dos gêneros Toxocara e Ancylostoma nestes ambientes representa um sério problema à saúde pública, uma vez que são agentes etiológicos da larva migrans visceral (LMV) e larva migrans cutânea (LVC), respectivamente (GUIMARÃES et al., 2005). A presença de LMV, principalmente em crianças, pois podem levar a lesão pulmonar, cerebral e hepática (PERUCA et al., 2009) e manifestações neurológicas como meningite eosinofílica, encefalite, mielite, vasculite cerebral e neurite óptica são relatadas (FERREIRA, 2012). Embora não seja fatal em humanos, a LMV se caracteriza por alterações pulmonares, hepatomegalia e eosinofilia, no qual o diagnóstico clínico pode estar relacionado à queixa do paciente em perda de apetite, febre, fadiga e dor abdominal. Para o diagnóstico epidemiológico, na anamnese deve ser investigada a presença de cães jovens não desverminados na residência, bem como o local que as crianças frequentam (DAMIAN et al., 2007).

Esta investigação objetivou verificar a contaminação de solo de praças públicas por parasitos de importância zoonótica na cidade de Lages, Santa Catarina.

\section{MATERIAL E MÉTODOS}

A cidade de Lages está localizada na região serrana de Santa Catarina (SC) e região sul do Brasil. As coordenadas geográficas são: latitude sul de $27^{\circ} 48^{\prime} 57^{\prime \prime}$ e longitude oeste de $50^{\circ} 20^{\prime} 33^{\prime \prime}$ e uma altitude de 916 metros; o clima é subtropical e temperatura média de $14,3^{\circ} \mathrm{C}$. A população é de aproximadamente 156.727 habitantes, taxa de crescimento populacional de 1,38 e densidade populacional de 59,27 habitantes $/ \mathrm{km}^{2}$ (IBGE, 2010). Lages conta com 64 praças, destas, oito foram selecionadas em sorteio aleatório (Figura 1) definindo-se duas centrais: Praça Jonas Ramos e a Praça Motorista e seis praças da periferia: Praças Evandel Xavier Soares e Fraternidade (Bairro Copacabana), Praça Herasmo Furtado (Bairro Sagrado Coração de Jesus), Praça Iraí (Bairro Petrópolis), Praça Frei Rogério (Bairro Eugênio Floriani)e Praça Jânio Wolff (Bairro Gethal).

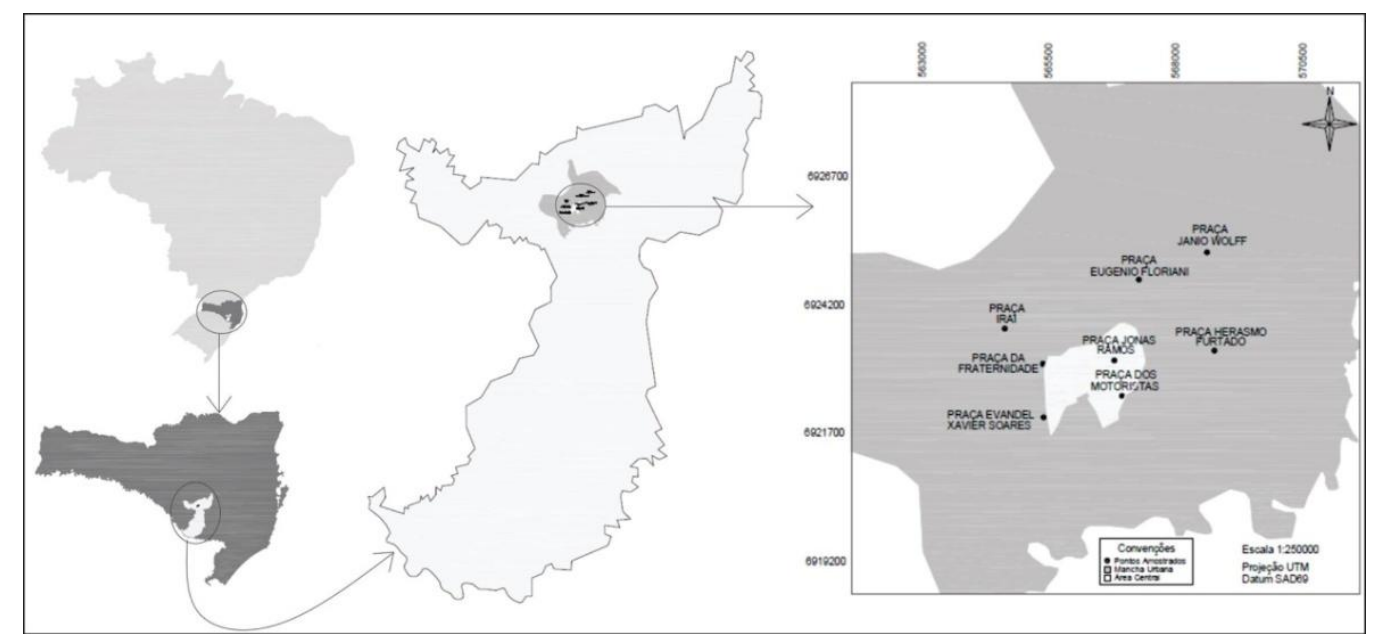

Figura 1 - Localização da cidade de Lages (SC) com destaque para as Praças Públicas amostradas.

O estudo foi conduzido de setembro de 2008 a abril de 2009. Foram estabelecidos três pontos de coleta para cada quadrante, a cada $9 \mathrm{~m}^{2}$ dentro de um quadrante $2 \times 2 \mathrm{~m}$. Em cada ponto foram recolhidas três amostras de solo, com ou sem material fecal a no máximo $2 \mathrm{~cm}$ da superfície. A periodicidade foi de uma coleta por estação. O pool de cada amostra foi acondicionado em saco plástico limpo, etiquetado e identificado. As amostras foram estocadas em isopor com gelo e encaminhadas ao Laboratório de Parasitologia da Universidade do Planalto Catarinense (UNIPLAC) e processadas num período máximo de 24 horas. O diagnóstico parasitológico foi baseado na técnica de sedimentação espontânea (HOFFMAN et al., 1934) para pesquisa de ovos e larvas, sob microscopia óptica com objetiva de 10X. Para análise estatística foi usado o teste não paramétrico de Qui-quadrado para tendências, adotado p<0,05 como nível de significância. 


\section{RESULTADOS}

Das 1602 amostras de solo analisadas (504 das praças centrais e 1098 das praças de bairros) ao longo de três estações do ano (primavera, verão e outono) nas praças públicas do município de Lages somente foram encontrados ovos de Toxocara spp., representando $0,75 \%(12 / 1602)$ de positividade. O teste Qui-quadrado não apontou diferença significativa $(\mathrm{p}=$
0,008) entre as proporções de amostras parasitadas nas áreas periféricas e centrais ou por estação do ano. Estes bairros apresentam grande variação em relação ao desenvolvimento econômico e infraestrutura, podendo repercutir principalmente em relação ao número de animais errantes que circulam entre ruas e praças. $\mathrm{O}$ resultado em relação às estações do ano e a localização das praças são mostrados na Tabela 1 .

Tabela 1 - Frequências (F) e percentagem (\%) de amostras positivas para ovos de Toxocara spp., coletadas em três estações de ano, em praças do município de Lages (SC), de 2008 a 2009.

\begin{tabular}{|c|c|c|c|c|c|c|c|c|c|}
\hline \multirow{2}{*}{$\begin{array}{l}\text { Localização das } \\
\text { Praças }\end{array}$} & \multirow{2}{*}{$\mathrm{n}$} & \multicolumn{2}{|c|}{ Primavera } & \multicolumn{2}{|c|}{ Verão } & \multicolumn{2}{|c|}{ Outono } & \multicolumn{2}{|c|}{ Total } \\
\hline & & $\mathrm{F}$ & $\%$ & $\mathrm{~F}$ & $\%$ & $\mathrm{~F}$ & $\%$ & $\mathrm{~F}$ & $\%$ \\
\hline Central & 504 & 4 & 0,79 & 1 & 0,20 & 1 & 0,20 & 6 & 1,19 \\
\hline Periférica & 1098 & 3 & 0,27 & 3 & 0,27 & 0 & 0,00 & 6 & 0,55 \\
\hline Total & 1602 & 7 & 0,44 & 4 & 0,25 & 1 & 0,06 & 12 & 0,75 \\
\hline
\end{tabular}

\section{DISCUSSAO}

As prevalências de contaminação de areia por parasitos zoonóticos em áreas públicas no Brasil, apresentando amplitude de zero até $100 \%$. A amostragem varia enormemente, pautado, talvez, pela proporção de praças de cada localidade, pelo tamanho da área e/ou a sua popularidade, bem como a preferência em levar os animais de companhia a defecar nas áreas públicas próximas de suas residências. Alguns trabalhos priorizaram a coleta de fezes deixadas em ambientes públicos e outros dão preferência pela coleta de areia. Fatores como protocolo de amostragem, técnica de diagnóstico, distribuição geográfica e principalmente à idade dos animais podem influenciar os resultados (GUIMARÃES et al., 2005; MELLO et al., 2011).

As amostras das praças de Lages mostraram prevalência baixa $(0,75 \%)$ para ovos de Toxocara $\mathrm{spp}$., entretanto, negatividade para este ascarídeo foi obtida em Juiz de Fora (MG), na amostragem de 16 praças públicas (SOUZA et al., 2008) e em 10 praças de Cuiabá (SOUSA et al., 2010). A negatividade e/ou a baixa prevalência de ovos de Toxocara spp. pode ser explicada pela baixa infecção deste parasito em animais adultos e a faixa etária dos cães que mais tem acesso às áreas públicas, também justificado no estudo de Nunes et al. (2000) em areia de praças de lazer em São Paulo, com prevalência zero ou baixa, referenciando principalmente a idade adulta dos animais que transitam nestes locais. Em relação à contaminação por ovos de Toxocara spp. pelo zoneamento da praça, Santarém et al. (2010) observaram que a contaminação foi maior nas praças na região periférica em relação as praças centrais da cidade de Paranapanema (SP), divergente desta investigação.

Prevalências de até $30 \%$ para ovos de Toxocara spp. em praças públicas foram registradas em: 1,3\% no município de Espírito Santo do Pinhal (SP) (SANTOS et al., 2003); 5\% em praças do município de Uruguaiana (RS) (GINAR et al., 2006); 0,8\% no bairro Ipanema, em Porto Alegre (RS) (MATESCO et al., 2006); 20,69\% em praças do município de Laguna (SC) (BLAZIUS et al., 2006); 4,32\% em praças públicas de Curitiba (PR) (LEITE et al., 2004); $30 \%$ em Santa Maria (RS) (OLIVEIRA et al., 2007); 3,3\% de positividade no Rio de Janeiro, Niterói e Teresópolis (BRENNER et al., 2008); 7,6\% em Anápolis (GO) (FRANCISCO et al., 2008); $25 \%$ em Duque de Caxias (RJ) (THOMÉ et al., 2008); 29,03\% em solo de assentamento rural em Pontal do Paranapanema (SP) (SANTARÉM et al., 2008) e 24,1\% em Pelotas (RS) (VILLELA et al., 2009).

Prevalências altas foram registradas em Santa Maria (CORRÊA \& MOREIRA, 1996) e Sorocaba (SP) (COELHO et al, 2001), respectivamente com 93,3\% e $53,3 \%$. Em 2004, a prevalência foi de $77 \%$ em praças públicas na região urbana da capital gaúcha (MENTZ et al., 2004); em Lavras (MG) 69,6\% das amostras de areia de praças públicas foram positivas para ovos de Toxocara spp. (GUIMARÃES et al. 2005); em Salvador (BA) a prevalência foi de 45,9\% (SANTOS et al., 2006) e de $71 \%$ em Uberlândia (MG)(ARAÚJO et al., 2008). Na zona sul urbana do Rio de Janeiro a positividade foi de $100 \%$ das amostras de areia das praças investigadas (SOUZA et al., 2008); em Canoinhas (SC), $100 \%$ das amostras de areia apresentaram ovos de Toxocara spp. e Ancylostoma spp. (PEDRASSANI et al., 2008); em Paranapanema (SP) foi registrado $76,9 \%$ de contaminação por ovos de Toxocara spp. (SANTARÉM et al., 2010) e na capital paulista a prevalência variou de $63,7 \%$ até $85,2 \%$ em praças públicas (SILVA et al. 2010; MELLO et al., 2011; ROCHA et al., 2011). As diferenças encontradas nos diversos estudos podem ter ocorrido devido às 
diversas metodologias empregadas ou mesmo refletir as diferenças entre os números de cães errantes entre as localidades, corroborado pelas conclusões de Araújo et al. (1999). O livre acesso de cães e gatos que defecam em áreas públicas e locais de lazer facilita a contaminação do solo. A presença de ovos de helmintos eliminados com as fezes, podem permanecer viáveis por longo período de tempo no ambiente que dependendo da temperatura e umidade levam a população ao risco de infecção e consequentemente levar a parasitoses, principalmente em crianças (ALDERETE et al., 2003; ANARUMA FILHO et al., 2003).

Estudo mais abrangente, envolvendo as diferenças socioeconômicas foi realizado em diferentes bairros da área urbana do município de Duque de Caxias (RJ) (THOMÉ et al., 2008). Apesar disso, observaram que mais da metade das praças visitadas estavam contaminadas por um ou dois parasitos (Ancylostoma spp. e Toxocara spp.). A percepção acerca dos locais de coleta em Lages evidenciou diferenças socioeconômicas nos diversos bairros, com prevalência da classe média na região central do município, embora não se possa, neste caso, associar uma frequência maior em razão da classe social, mas em relação ao volume de pessoas e animais que transitam e utilizam as praças centrais do município, objeto de eventos municipais.

Este estudo foi conduzido durante três estações do ano: primavera, verão e outono, com prevalência maior na primavera $(0,44 \%)$ em comparação com verão $(0,25 \%)$ e outono $(0,06 \%)$. Comparando as condições climáticas na recuperação de ovos de geohelmintos, em amostras de solo de praças da zona leste da cidade de São Paulo (SILVA et al., 2010; MELLO et al., 2011), os gêneros Ascaris e Toxocara foram os mais prevalentes e a contaminação ocorreu em qualquer época do ano. Mello et al. (2011) evidenciaram um percentual maior (51,2\%) na estação chuvosa e com temperatura ambiental mais alta; e Ginar et al. (2006) não demonstraram diferença significativa entre as praças do município de Uruguaiana (RS) em relação às estações do ano e Araújo et al. (2008) não encontraram diferença significativa para ovos de Toxocara spp. na estação seca $(88 \%)$ e chuvosa (71\%), porém, em Maringá (PR) Toxocara spp. foi o helminto mais frequentemente observado em praças públicas, com ocorrência de $95,38 \%$ no inverno e de $69,23 \%$ no verão (TIYO et al., 2008).

Em relação ao tipo de solo, as praças da cidade de Lages, apresentam uma variação entre solo arenoso observado principalmente na praça central e argiloso encontrados nas praças da periferia. Para Rocha et al. (2011), o solo arenoso constitui um relevante foco de infecção humana por parasitos, devido a sua característica geológica que permite reter água na estrutura porosa do solo. Os estágios de ovos e larvas de parasitos necessitam para sua sobrevivência não só da temperatura mas também a umidade que pode ser assegurada por este tipo de solo. Os ovos de Ascarídeos se desenvolvem bem em solos argilosos, concentrando-se, por ação das chuvas, em local abaixo da superfície (ovos ou larvas) (OPS, 2003).

Neves \& Massara (2009) afirmaram que os levantamentos sobre a contaminação do solo são fundamentais para se conhecer o nível de exposição a LMV que a população está sujeita. Esses levantamentos devem estar sempre associados a ações educativas visando o controle dessas enfermidades. Por isso a necessidade de sensibilizar a população em manter uma posse responsável de seus animais, juntamente com o controle populacional dos cães de rua e a necessidade de orientações feitas pelos médicos veterinários para o controle parasitário de seus pacientes. Também é importante a apreensão de animais vadios e a construção de cercas ao redor das áreas de recreação, como caixas de areia ou sua cobertura com lonas durante a noite. A realização de programas integrados de educação sanitária, visando ampliar os conhecimentos dos professores de educação infantil sobre os aspectos relacionados à aquisição de zoonoses deve ser questionada. Outro fator relevante no controle das referidas zoonoses refere-se à educação dos proprietários de cães e gatos, evitando o acúmulo de seus dejetos em ambientes públicos e o acesso a centros educacionais, além da administração periódica de anti-helmínticos sob a orientação de médicos veterinários (SOUSA et al., 2010).

Esta investigação detectou somente a contaminação de areia de praças públicas da cidade de Lages por ovos de Toxocara spp. não ocorrendo diferença significativa entre as praças da periferia ou as centrais estudadas e entre as estações do ano. Embora a prevalência de ovos tenha sido baixa, se faz necessário medidas mais efetivas de sensibilização da população sobre a saúde dos animais de companhia evitando assim o risco de infecção humana.

\section{CONFLITO DE INTERESSE}

Os autores deste estudo afirmam não apresentar nenhum tipo de conflitos de interesse.

\section{REFERÊNCIAS}

ALDERETE, J. M. S.; JACOB, C. M. A.; PASTORINO, A. C.; ELEFANT, G. R.; CASTRO, A. P. M.; FOMIN, A. B. F.; CHIEFFI, P. P. Prevalence of Toxocara infection in schoolchildren from the Butantã Region, São Paulo, Brazil. Memórias do Instituto Oswaldo Cruz, v.98, n.5, p.593-597, 2003.

ANARUMA FILHO, F. A; CHIEFFI, P. P.; CORREA, C. R. S.; CAMARGO, E. D.; SILVEIRA, E. P. R.; ARANHA, J. J. B. Human Toxocariasis: incidence among residents in the outskirts of Campinas, State of São Paulo, Brazil. Revista do Instituto de Medicina Tropical São Paulo, v.45, n.5, p.293-294, 2003.

ARAÚJO, F. R.; RODRIGUES, R. G.; CAVALHAES, J.; SMIYOSHI, M. I.; SALGADO, F. P.; SILVA, M. A.; PEREIRA, M. L. Contaminação de praças públicas de Campo Grande, Mato Grosso do Sul, Brasil, por ovos de Toxocara e Ancylostoma em fezes de cães. Revista da Sociedade Brasileira de Medicina Tropical, v.32, n.5, p.581-583, 1999. 
ARAÚJO, N. S.; RODRIGUES, C. T.; CURY, M. C. Helmintos em caixas de areia em creches da cidade de Uberlândia, Minas Gerais. Revista de Saúde Pública, v.42, n.1, p.150-153, 2008.

BLAZIUS, R. D. ; SANTOS, O. S.; KAULING, A. L.; RODRIGUES, D. F. P.; LIMA, M. C. Contaminação da areia do Balneário de Laguna, SC, por Ancylostoma spp. e Toxocara spp. em amostras fecais de cães e gatos. Arquivo Catarinense de Medicina, Santa Catarina, v.3, n.3, p.55-58, 2006.

BRENNER, B.; MATTOS, D. P. B. G.; MILLAR, P. R.; ARASHIRO, E. K. N.; DUQUE-FERREIRA, V.; SUDRÉ, A. P. Estudo da contaminação de praças públicas de três municípios do Estado do Rio de Janeiro, Brasil, por ovos e larvas de helmintos. Revista de Patologia Tropical, v.37, n.3, p.247-254, 2008.

COELHO, L. M. P. S.; DINI, C. Y.; MILMAN, M. H. S. A.; OLIVEIRA, S.M. Toxocara spp. eggs in public squares of Sorocaba, São Paulo State, Brazil. Revista do Instituto de Medicina Tropical São Paulo, v.43, n.4, p.189-191, 2001.

CORRÊA, G. L. B.; MOREIRA, W. S. Contaminação do solo por ovos de Ancylostoma spp. em praças públicas de Santa Maria, RS, Brasil. Revista da Faculdade de Zootecnia, Veterinária e Agronomia, Bagé, v.2/3, n.1, p.18-23, 1996.

DAMIAN, M. M.; MARTINS, M.; SARDINHA, J. F.; SOUZA, L. O.; CHAVES, A.; TAVARES, A. M. Frequência de anticorpo anti-Toxocara canis em comunidade do Rio Uatumã, no Estado do Amazonas. Revista do Instituto de Medicina Tropical São Paulo, v.40, n.6, p.661-664, 2007.

FERREIRA, M. U. Parasitologia contemporânea. Rio de Janeiro: Guanabara Koogan, 2012. p.124.

FRANCISCO, M. M. S.; SILVA, R. C. Prevalência de ovos e larvas de Ancylostoma spp. e de Toxocara spp. em praças publicas da Cidade de Anápolis - GO. Ensaios e Ciência: Ciências Biológicas, Agrárias e Saúde, v.12, n.1, p.131-137, 2008.

GUIMARÃES, A. M.; GABELLINI, E.; ALVES, L.; REZENDE, G. F.; RODRIGUES, M. C. Ovos de Toxocara sp. e larvas de Ancylostoma sp. em praça pública de Lavras, Minas Gerais. Revista de Saúde Pública, v.39, n.2, p.293-295, 2005.

GINAR, R. M. B.; GALARÇA, R. C. G.; PICAVÊA, J. P.; PETRY, H. Índice de contaminação do solo por ovos dos principais nematóides de caninos nas praças públicas da cidade de Uruguaiana - RS, Brasil. Revista da Faculdade de Zootecnia, Veterinária e Agronomia, v.13, n.1, p.103-111, 2006.

HOFFMAN, W. A.; PONS, J. A.; JANER, J. L. The sedimentation concentration method in Schistosomiasis mansoni. Puerto Rico Journal of Public Health, v.9, p.283-298, 1934.

INSTITUTO BRASILEIRO DE GEOGRAFIA E ESTATÍSTICA - IBGE - Fundação Sistema Estadual de Análise de Dados. Censo Demográfico: Santa Catarina; 2010. Disponível em:< http://www.ibge.gov.br/cidadesat/topwindow.htm?1>. Acesso em: 10/01/12.

LEITE, L. C.; CIRIO, S. M.; DINIZ, J. M. F.; MARINONI, L. P.; SILVA, A. W. C.; LUZ, E.; VARGAS, C. C. S. G.; LEITE, S. C.; ZADOROSNEI, A. C. B.; VERONESI, E. M.; BARRANCO, R. Contaminação por ovos de Toxocara spp. em praças públicas e parques recreacionais (jardinetes) de Curitiba - Paraná - Brasil. Revista Acadêmica: Ciências Agrárias e Ambiental, v.2, n.2, p.59-64, 2004.

MATESCO, V. C.; MENTZ, M. B.; ROTT, M. B.; SILVEIRA, C. O. Contaminação sazonal por ovos de helmintos na praia de Ipanema, em Porto Alegre, Rio Grande do Sul, Brasil. Revista de Patologia Tropical, v.35, n.2, p.135-141, 2006.

MELLO, C. S.; MUCCI, J. L. N.; CUTOLO, S. A. Contaminação parasitária de solo em praças públicas da zona leste de São Paulo, SP - Brasil e a associação com variáveis meteorológicas. Revista de Patologia Tropical, v.40, n.3, p.253-262, 2011.

MENTZ, M. B.; ROTT, M. B.; JACOBSEN, S. I. V.; BALDO, G.; RODRIGUES-JÚNIOR, V. Frequency of Toxocara spp. in three public parks of Porto Alegre city - Brazil. Revista de Patologia Tropical, v.33, n.1, p.105-112, 2004.

NEVES, R. L. S.; MASSARA, C. L. Contaminação do solo de áreas comunitárias do município de Caratinga, MG, Brasil, por ovos de Toxocara sp. e cistos de Entamoeba sp. Revista de Patologia Tropical, v.38, n.2, p.126-130, 2009.

NUNES, C. M., PENA, F. C.; NEGRELLI, G. B.; ANJO, C. G. S., NAKANO, M. M.; STOBBE, N. S. Ocorrência de larva migrans na areia de áreas de lazer das escolas municipais de ensino infantil, Araçatuba, SP, Brasil. Revista de Saúde Pública, v.34, n.6, p.656-658, 2000.

OLIVEIRA, C. B.; SILVA, A. S.; MONTEIRO, S. G. Occurrence of parasites in soils of childish squares in day care centers municipality of Santa Maria (RS), Brazil. Revista da Faculdade de Zootecnia, Veterinária e Agronomia, v.14, n.1, p.174-179, 2007.

ORGANIZAÇÃO PANAMERICANA DE SAÚDE OPS/OMS. 2003. Helmintiasis Intestinales. Manejo de las Geohelmintiasis. Departamento de Parasitología y Micología, Instituto de Higiene, Facultad de Medicina, Universidad de la República, Uruguay. 39p. 
PEDRASSANI, D.; VIERA, A. M.; THIEM, E. M. B. Contaminação por Toxocara spp. e Ancylostoma spp. em áreas de lazer do município de Canoinhas, SC. Archives of Veterinary Science, v.13, n.2, p.110-117, 2008.

PERUCA, L. C. B.; LANGONI, H.; LUCHEIS, S. B. Larva migrans visceral e cutânea como zoonoses: revisão de literatura. Veterinária e Zootecnia, v.16, n.4, p.601-616, 2009.

ROCHA, S., PINTO, R. M. F., FLORIANO, A. P., TEIXEIRA, L. H., BASSILI, B., MARTINEZ, A., COSTA, S. O. P. DA \& CASEIRO, M. M. Environmental analyses of the parasitic profile found in the sandy soil from the Santos municipality beaches, SP, Brazil. Revista do Instituto de Medicina Tropical São Paulo, v.53, n.5, p.277-281, 2011.

SANTARÉM, V. A.; DIAS, A. P.; FELIX, A.; RODENAS, R. S.; SILVA, A. V. Contaminação por ovos de Toxocara spp. em praças públicas das regiões central e periurbana de Mirante do Paranapanema, São Paulo, Brasil. Veterinária e Zootecnia, v.17, n.1, p.4753, 2010.

SANTARÉM, V. A.; FRANCO, E. C.; KOZUKI, F. T.; FINI, D.; PRESTES-CARNEIRO, L. E. Enviromental contamination by Toxocara spp. eggs in a rural settlement in Brazil. Revista do Instituto de Medicina Tropical São Paulo, v.50, n.5, p.279-281, 2008.

SANTOS, R. S.; BONATO, L. C.; MARQUES, M. P. A. Ocorrência de agentes causais de larva migrans em areias destinadas à recreação em escolas de ensino infantil em Espírito Santo do Pinhal, SP, Brasil. Revista Ecossistema, São Paulo, v.28, n.1-2, p.57-60, 2003.

SANTOS, N. M.; SILVA, V. M. G.; THÉ, T. S.; SANTOS, A. B.; SOUZA, T. P. Contaminação das praias por parasitos caninos de importância zoonótica na orla da parte alta da cidade de Salvador, BA. Revista de Ciências Médicas e Biológicas, v.5, n.1, p.40-47, 2006.
SCAINI, C. J. ; TOLEDO, R. N.; LOVATEL, R.; DIONELLO, M. A.; GATTI, F. A.; SUSIN, L., SIGNORUNI, V. R. M. Environment contamination by helminth eggs and larvae in dog feces from central area of Cassino Beach, Rio Grande do Sul. Revista da Sociedade Brasileira de Medicina Tropical, v.36, n.5, p.617-619, 2003.

SILVA, C.; MUCCI, J. L.; CUTOLO, S.; SANTOS, J.; SOUSA, T.; MARTINS, C.; CASSENOTE, A. Efeitos da variação climática na contaminação do solo de praças públicas por parasitas na zona leste de São Paulo. Revista Saúde, Santa Maria, v.4 (Esp.1), p.1, 2010.

SOUZA, F. D. , MAMEDE-NASCIMENTO, T. L. ; SANTOS, C. S. Encontro de ovos e larvas de helmintos no solo de praças públicas na zona sul da cidade do Rio de Janeiro. Revista de Patologia Tropical, v.36, n.3, p.247-253, 2008.

SOUSA, V. R., ALMEIDA, A. F.,CÂNDIDO, A. C.; BARROS,L. A. Ovos e larvas de helmintos em caixas de areia de creches, escolas municipais e praças públicas de Cuiabá, MT. Ciência Animal Brasileira, v.11, n.2, p.390-395, 2010.

TIYO, R., GUEDES, T. A., FALAVIGNA, D. L. M. ; FALAVIGNA-GUILHERM, A. L. Seasonal contamination of public squares and lawns by parasites with zoonotic potential in southern Brazil. Journal of Helminthology, v.82, n.1, p.1-6, 2008.

THOMÉ, S. M., LAFAYETTE, E.P., PESSOA NETO, G.R. Contaminação ambiental por ovos de Toxocara spp. e Ancylostoma spp. em praças públicas no Município de Duque de Caxias. Saúde \& Ambiente, Rio de Janeiro, v.3, n.2, p.30-32, 2008.

VILLELA, M. M.; PEPE, M. S.; FERRAZ, M. L.; MORAIS, N. C. M.; ARAÚJO, B. A.; RUAS, J. L.; MÜLLER, G.; BERNE, M. E. A. Contaminação ambiental da orla da Laguna dos Patos (Pelotas, RS, Brasil), por parasitos com potencial zoonótico. Vittalle, Rio Grande, v.21, n.2, p.69-74, 2009. 